\title{
Propiedades Psicométricas: Escala Yucatán del Uso Percibido de la Inteligencia Emocional en Menores (EYUPIE-M)
}

\author{
Psychometric Properties: Yucatan Scale of Perceived Use of Emotional Intelligence \\ in Minors (EYUPIE-M)
}

Manuel Sosa-Correa ${ }^{1}$, Agustín Rodríguez-Ake ${ }^{2}$ Ricardo Castillo Ayuso ${ }^{3}$, Nicolás Ponce ${ }^{4}$ y José M.
Mestre $^{5}$

\begin{abstract}
Resumen
Debido a que la inteligencia emocional se vincula con las experiencias significativas en las personas (Mayer, Caruso, \& Salovey 2016), se busca convertir la EIIE (Sosa-Correa \& Rodríguez-Ake, 2011) a un nuevo instrumento (EYUPIE-M) que evalúe el uso que los menores de edad le dan a su inteligencia emocional percibida (IEP) y conocer la naturaleza y significación de la relación de este nuevo instrumento con otros de IEP como el TMMS24, el ESCQ y con el PANAS. La Escala Yucatán del uso percibido de la IE en menores (EYUPIE-M; fiabilidad alfa $=.87)$ consta de 24 ítems y cuatro sub escalas: Conciencia Emocional Intrapersonal (CE-Intra), Uso Prosocial (UPS), Conciencia Emocional Interpersonal (CE-Inter) y Uso Antisocial (UAS). La consistencia interna de las sub-escalas varió entre .72 y .85. Solo se encontró que CEIntra, UPS y CE-Inter se relacionaron positivamente con las tres sub-escalas del TMMS24, el UPS con todas las del ESCQ y el UAS negativamente con reparación del TMMS24 y con regulación del ESCQ.
\end{abstract}

Palabras clave: inteligencia emocional percibida, menores de edad, funcionamiento prosocial, funcionamiento antisocial

\begin{abstract}
Because emotional intelligence is linked to meaningful experiences in people (Mayer, Caruso, \& Salovey 2016), it seeks to convert the EIIE (Sosa-Correa \& Rodríguez-Ake, 2011) to a new instrument (EYUPIE-M) to evaluate the use that minors give their perceived emotional intelligence (IEP) and to know the nature and significance of the relationship of this new instrument with other IEPs such as TMMS24, ESCQ and PANAS. The Yucatecan Scale of IEP perceived use in minors (EYUPIE-M, alpha reliability $=.87$ ) consists of 24 items and four sub-scales: Intrapersonal Emotional Consciousness (CE-Intra), Prosocial Use (UPS), Interpersonal Emotional Consciousness CE-Inter) and Antisocial Use (UAS). The internal consistency of the subscales ranged from .72 to .85 . It was found that only CE-Intra, UPS and CE-Inter were positively related to the three sub-scales of the TMMS24, and so the UPS with all of the ESCQ and UAS, negatively with TMMS24 repair and with ESCQ regulation.
\end{abstract}

Keywords: perceived emotional intelligence, minors, prosocial functioning, and antisocial functioning

\footnotetext{
${ }^{1}$ Dr. En Ciencias Cognitivas. Universidad Autónoma de Yucatán. Profesor Titular. Dirección Postal: Kilometro 1 Carretera Mérida Tízimin-Cholul C.P. 97305. Tel.: +52 (999) 9432045, y 9433888. Correo: mscorrea@ correo.uady.mx

${ }^{2}$ Maestro en Ciencias, Universidad Marista de Yucatán, Docente titular, Periférico Norte Tablaje Catastral 13941, Carretera Mérida Progreso, Sin Nombre de Col 27, 97300 Mérida, Yuc., Tel.:+521 9992364538. Correo: agustin.ake@ gmail.com

${ }^{3}$ Mtro. en Psic. Educ. Universidad Autónoma de Yucatán. Coordinador de la salida terminal en Psicología Clínica para adultos, de la maestría en psicología aplicada. Dir Postal: Facultad de Psicología de la UADY, K 1 Carretera Mérida, Tizimín Cholul. CP 97305. +52(999)9473794, ricastil@correo.uady,mx

${ }^{4}$ Doctor en Educación, Departamento de Métodos de Investigación y Diagnóstico en Educación, Facultad de Ciencias de la Educación, Universidad de Granada, Dir. Postal: Campus Universitario de Cartuja C.P. 18071 Granada (Granada), Despacho 314.

${ }^{5}$ Doctor en Psicología, Universidad de Cádiz, Profesor Titular de Universidad, Departamento de Psicología, Facultad de Ciencias de la Educación, Campus Puerto Real, 11519, España. +34 956016220; josemi.mestre@uca.es
} 


\section{Introducción}

Recientemente el concepto de inteligencia emocional (IE) acaba de cumplir sus primeros 25 años desde que Salovey y Mayer (1990) lo definieron por vez primera como un conjunto de capacidades para el procesamiento de la información emocional que provea un adecuado funcionamiento personal y social. Con motivo de dicho aniversario la revista Emotion Review ha realizado un monográfico sobre el "estado del arte" de esta estructura de trabajo denominada Inteligencia Emocional. A tenor de la conclusión de los editores del monográfico, no existen dudas de que el tópico de la IE ha florecido y desarrollado (Barchard, Brackett, \& Mestre, 2016).

No obstante, quedan algunas cuestiones relevantes por atender. Especialmente las relacionadas con la evaluación de la IE y con la diatriba entre las dos visiones teóricas predominantes del concepto (Roberts, MacCann Guil \& Mestre, 2016). Actualmente, existen dos aproximaciones teóricas sobre la IE. Por un lado, el modelo de la IE como capacidad. Basado en el concepto original de 1990 (Salovey \& Mayer, 1990), y en su desarrollo posterior de 1997 (Mayer \& Salovey, 1997) que recientemente ha progresado a su versión 3.0 con una declaración de principios y su actualización (Mayer, Caruso, \& Salovey, 2016). Por otro lado, la visión de la IE como rasgos de personalidad (para una revisión actualizada véase Petrides, 2016) que visualiza la IE como un perfil de rasgos de personalidad que propician el desarrollo de comportamientos emocionalmente inteligentes.

Algunos autores han defendido que estas dos conceptualizaciones no compiten entre sí necesariamente, sino que podrían ser complementarias (Mestre, MacCann, Guil, \& Roberts, 2016) porque se necesita tanto de una adecuada creencia en las capacidades emocionales como el hecho de poseerlas (Gutentag, Halperin, Porat, Bigman, \& Tamir, 2016). La activación de cualquier estrategia de regulación requiere saber qué hacer y una tendencia a hacerlo, por tanto, el estudio de la IE debería incluir la habilidad y el rasgo (Roberts, MacCann, Guil, \& Mestre, 2016).

Otro aspecto que se ha señalado relevante es que aún no hay estudios sobre la IE como variable criterio y no sólo como una variable predictiva (Roberts et al., 2016) ya que la IE crece y se desarrolla con la edad; por consiguiente, deben existir ciertos factores que influyan o inhiban su potencial de desarrollo.

IE se vincula con las experiencias que tienen un significado para la vida, y no con situaciones artificiales o distantes a las experiencias propias de la persona (Mayer et al., 2016). Cuando procesamos cognitivamente estas experiencias emocionales con significado tanto existencial como experiencial se denomina información hot. Por el contrario, cuando lo que se procesa es información basada en estímulos sin significado emocional, como una simple suma de números, se denomina información cool (Gutiérrez-Cobo, Cabello, \& Fernández-Berrocal, 2016).

En la revisión del concepto de IE como capacidad, se ha postulado que la IE es un conjunto de cuatro capacidades (percibir y expresar, facilitar, comprender y regular emociones) especializadas en el tratamiento de la información emocional hot (pues las emociones tienen un componente basado en la valoración de las personas), mientras que otros tipos de inteligencias estarían más orientadas al tratamiento de la información cool, por ejemplo, en el razonamiento abstracto (Mayer et al., 2016). El procesamiento de la información hot, como la emocional, y el funcionamiento personal y social están conectados (Gutiérrez-Cobo et al., 2016) y se relaciona una alta capacidad en IE con adecuados niveles de bienestar mental y bajos niveles de síntomas depresivos (Sánchez-Álvarez, Extremera, \& Fernández-Berrocal, 2016), con un buen funcionamiento laboral (Lopes, 2016) y con adecuados niveles de adaptación escolar y bajos niveles de acoso escolar (Nathanson, Rivers, Flynn, \& Brackett, 2016).

Por otro lado, desde el modelo de rasgos de la IE, se ha señalado que la IE se encuentra íntimamente conectada con el funcionamiento personal y social así como con la calidad de vida (Petrides et al., 2016). Por ejemplo, algunos trabajos han señalado cómo ciertas muestras clínicas con un escaso funcionamiento social como las denominadas "tríadas oscuras de la personalidad" (narcisismo, maquiavelismo y psicopatología subclínica, véase Paulhus \& Williams, 2002) podría tener una clara 
explicación en el mal uso que hacen tanto de sus propias capacidades de IE como de sus tendencias a ser socialmente ineficaces y dañinos (Austin, Farrelly, Black, \& Moore, 2007; Petrides, Vernon, Schermer, \& Veselka, 2011). En este sentido Dufey y Fernandez (2012) muestran que la generación de malestar hacia otros está significativamente correlacionado con el Afecto Negativo y en correlación inversa con Afecto Positivo.

Por consiguiente, uno de los pasos necesarios a dar para continuar el progreso de la investigación en IE, es el desarrollo de medidas para observar no solo la percepción que las personas tienen de su IE (aunque ya hay muchas medidas con dicho objetivo) sino del uso de dichas capacidades de IE encaminadas a su funcionamiento.

Por tres razones es necesario construir o diseñar escalas para medir con qué finalidad usan las personas su IE: La primera, ya comentada, es que IE es una capacidad que se desarrolla con la edad, algo que defienden ambas perspectivas de la IE (Extremera, Fernández-Berrocal, \& Salovey, 2006; Fariselli, Ghini, \& Freedman, 2008) por lo tanto, es un tipo de inteligencia cristalizada que, se desarrolla con la edad por las influencias de las experiencias vividas y del conocimiento emocional adquirido fundamentalmente (Mestre, Núñez-Vázquez, \& Guil, 2007). Segunda, algunos autores han indicado que la IE es una de las variables claves para un buen funcionamiento personal y social, pero no hay la claridad en saber que motivan a las personas a realizar determinados usos de la IE (Mestre, Guil, Rodriguez-Cordón, Pérez-González, \& Cejudo, 2015) Es decir, aún se desconoce bien por qué hay personas que usan la IE con fines antisociales (maquiavelismo) o con fines prosociales (Roberts et al., 2016). Como consecuencia de lo anterior, la tercera, es que la razón de este desconocimiento puede estar más en el interés de los investigadores en saber qué predice la IE sobre otros criterios como el funcionamiento personal o social (estudios de validez predictiva), en vez de querer saber qué factores psicológicos hacen florecer y desarrollar la IE (usar la IE como variable criterio).

Por consiguiente, un primer paso es desarrollar medidas que nos informen sobre la percepción que las personas hacen del uso de su IE, es decir, con qué finalidad utilizan su IE.

El primer objetivo de este trabajo es hacer un nuevo análisis a una medida de la IE (EIIE, Escala Infantil de la IE, Sosa-Correa \& Rodriguez-Ake, 2011) para desarrollar un instrumento que evalúe cómo los adolescentes usan su IE, tomando en cuenta las sub-escalas con fines antisociales y prosociales.

El segundo objetivo sería observar las relaciones del nuevo instrumento con otros de IE percibida (TMMS24 y el ESCQ) para ver el grado y signo de relación entre la nueva versión y las ya consolidadas (predictoras). Asimismo, también observar con la prueba del PANAS qué tipo de afectividad (positiva o negativa) podrían estar relacionada a las diferentes sub-escalas que se desarrollaran a partir de la nueva escala.

\section{Método}

\section{Participantes}

Por un proceso de muestreo incidental, se solicitó a varios centros de educación primaria y secundaria, privados y públicos del estado de Yucatán (México), la participación en esta investigación. En total 693 estudiantes participaron, con un rango de edad entre los $9 \mathrm{y}$ los 17 años de edad $(M=13.51 ; D T=1.45)$ de los cuales el $52.5 \%$ fueron mujeres.

Debido a las restricciones administrativas para la aplicación, por parte de las escuelas, los instrumentos para esta investigación fueron aplicados según el tiempo otorgado por las escuelas. Por lo tanto; del total anterior, solo 596 sujetos (el 86\% del total de la muestra) además de la EIIE contestaron el TMMS-24 (FernandezBerrocal, Extremera, \& Ramos, 2004), para su validación predictiva concordante y solo 78 participantes (14.99\% del total) completaron, las anteriores, el ESCQ (Faria et al., 2006) y el PANAS (Sandín, Chorot, Valiente \& Santed, 1998) para observar su relación con el afecto positivo o negativo.

\section{Procedimiento y cuestiones éticas}

Para la realización de este estudio, se contactó con una amplia muestra de centros de educación secundaria de Yucatán (México) solicitando su permiso para la investigación. De las que 
contestaron afirmativamente a esta solicitud, el equipo investigador visitó a la Dirección de los centros donde se les entregó un compromiso de confidencialidad de la información de los participantes. Así como la concreción de las fechas para la aplicación de las pruebas, resguardando los principios éticos de la investigación, debido a que los alumnos que contestaron las pruebas son menores de edad, se les pidió a sus padres que firmaran su autorización en un consentimiento informado en el que se mencionó que los datos recabados de usaran de manera anónima y para fines de investigación, todos los padres firmaron dicho consentimiento. Por su parte, los estudiantes respondieron a los instrumentos de manera voluntaria y anónima en el horario de tutoría.

Como contrapartida, se les mandó a las escuelas un informe general de los resultados por centro. En todo momento, se siguieron las diligencias éticas para este tipo de estudios al tratarse de menores.

\section{Instrumentos}

EIIE (Escala Infantil de Inteligencia Emocional, Sosa-Correa, \& Rodriguez-Ake, 2011)

La escala EIIE consta de 96 ítems y se construyó en función del modelo teórico de IE como capacidad (Mayer \& Salovey, 1997).

De acuerdo con Sosa-Correa y Rodríguez-Ake (2011), la EIIE siguió un proceso de validación de constructo, donde diez jueces expertos mostraban su grado de acuerdo o desacuerdo con los ítems sobre en cuál de los cuatro factores del modelo de Mayer y Salovey debería ser incluido dicho ítem (percepción, uso, comprensión y regulación). Cuando el grado de acuerdo entre jueces era, al menos, del $80 \%$, dicho ítem se consideraba perteneciente a dicho factor. Análisis factoriales confirmatorios comprobaron que algunos de estos ítems parecían pertenecer a un factor que se relacionaba con la idea de un uso antisocial de la IE, y la confirmación del modelo de IE de Mayer y Salovey, (1997) no se dio según las cuatro ramas que proponen.

La escala EIIE final era de 43 ítems. Esta escala consta de tres opciones de respuesta: 1 "no lo hago", 2 "a veces lo hago" y 3 "sí lo hago". En el trabajo de Sosa-Correa y Rodriguez-Ake (2011) se informó de una consistencia interna de 0.85 para la escala total. Un ejemplo de ítem para esta primera versión es: "Cuando no es apropiado que un amigo se sienta bien, ¿puedes hacer que cambie lo que siente?".

En la primera versión se aplicó a niños entre 9 y 17 años de edad, aunque la fiabilidad total era adecuada no se ajustaban a los cuatro factores inicialmente buscados y el hecho, relevante para el propósito de esta investigación, es que uno de los factores parecía indicar un "lado oscuro" (uso antisocial) en la IE. Esta primera versión contaba con un elevado número de ítems. Por lo tanto, un nuevo análisis permitiría reducir ítems y refactorizarla.

Trait Meta-Mood Scale (TMMS-24, versión en español de Fernandez-Berrocal et al., 2004)

El TMMS-24 consta de 24 ítems con 3 subescalas: Atención (At), Claridad (Cl) y Reparación emocional (Re); con 8 ítems cada una y con respuestas tipo Likert de 5 puntos ( $1=$ Nada de acuerdo a 5= Totalmente de acuerdo) que indica el grado de acuerdo respecto a los ítems. En este estudio, un total de 596 sujetos rellenaron el instrumento y la fiabilidad para cada una de las sub-escalas fue de $0.80(\mathrm{At}), 0.81(\mathrm{Cl})$ y $0.81(\mathrm{Re})$ siendo la fiabilidad total de la escala de 0.89 .

Esta escala de inteligencia emocional percibida (IEP) sirvió para ver la relación de concordancia con la EYUPIE-M. El TMMS-24 ha sido aplicado en trabajos similares en muestras de Chile y España (Fernández-Berrocal et al., 1998) con similares índices de consistencia interna al nuestro, así como en otros trabajos de validez predictiva del TMMS-24 (Díaz-Castela et al., 2013; Fernandez-Berrocal et al., 2004; Jiménez \& López Zafra, 2011), con sujetos entre 11 y 17 años teniendo como resultados de fiabilidad en At, $\alpha=.76 ; \mathrm{Cl}, \alpha=.85 ; \mathrm{y} \mathrm{Re}, \alpha=.65$.

El TMMS-24 ha mostrado ser bastante útil en los estudios de validez predictiva, especialmente la sub-escala de Reparación emocional, con criterios de bienestar mental y de rendimiento académico (Fernández-Berrocal \& Extremera, 2005).

\section{Emotional Skills and Competences Questionnaire (ESCQ, versión en español de Faria et al., 2006)}

Este instrumento consta de 45 items con 3 sub-escalas, Percepción y Comprensión 
Emocional (PCM), Expresión y Etiquetado de Emociones (EEE) y Manejo y Regulación de Emociones (MRE). En nuestro estudio con 78 sujetos las fiabilidades fueron respectivamente de:.71, .69 y .69. Siendo la fiabilidad total de la escala de .88 .

En un trabajo de Taksic, Mohoric y Duran (2009) con personas entre los 14 y 19 años, el ESCQ con una fiabilidad total de $\alpha=0.88$ mostró una correlación positiva y moderada entre las escalas que permitieron la composición lineal de una medida en general de competencia emocional con una confiabilidad de PCM entre $\alpha=.81$ y .90 , en EEE $\alpha=.78$ y .88 ; y con MRE $\alpha=.67$ y .78

El Positive and Negative Affect Schedule (PANAS versión en español de Sandín et al., 1998)

Es un listado de 20 sentimientos y emociones que se puntúan del 1 al 5 según el grado en el que el evaluado se siente. Se divide en dos escalas de 10 ítems cada una, una de afecto positivo (AP) y otra de afecto negativo (AN), más una tercera "Balance" que sale de la resta de la puntuación del AP y el AN. En el presente estudio con 78 sujetos las fiabilidades fueron respectivamente de: .81 (Total), .76 (AP) y .84 (AN).

En el estudio de Sandín et al., 1998), se realizó con estudiantes universitarios cuya edad promedio fueron de 20.47 años en mujeres y 20.59 años hombres años. La fiabilidad en hombres para el AP fue $\alpha=.89$ y la de AN $\alpha=.91$ y para las mujeres, $\mathrm{AP} \alpha=.87$ y $\mathrm{AN} \alpha=.89$

\section{Análisis estadísticos}

Para los fines de este estudio se utilizaron tres análisis factoriales (AF), un análisis de fiabilidad de consistencia interna con Alfa de Cronbach, y cálculos de correlaciones de Pearson. Primeramente se procedió al AF con extracción de máxima verosimilitud y sin rotación, con el objeto de excluir reactivos de baja carga factorial. Posteriormente, con un segundo AF de extracción de máxima verosimilitud y rotación varimax se buscó una estructura factorial más adecuada para los datos. A continuación, se estimaron los niveles de confiabilidad para la escala y se removieron reactivos que redujeran el valor de Alfa de Cronbach por debajo de los límites permitidos. El cuarto paso consistió en realizar otro AF para confirmar la estructura subyacente y se procedió a calcular correlaciones de Pearson entre cada uno de los factores generados con el TMMS24, el ESCQ, y el PANAS.

\section{Resultados}

Para el primer objetivo, se realizó un análisis exploratorio con todos los ítems del EIIE (SosaCorrea \& Rodriguez-Ake, 2011). Previo al análisis factorial, la escala EIIE de 43 ítems mostró buenos índices para su realización, en concreto, en la prueba KMO (Kaiser-MeyerOlkin) se obtuvo un índice de 0.94 , lo que sugiere que el tamaño muestral es adecuado para el AF. Por su parte la prueba de Barlett, el $\chi^{2}$ fue de $10708.881 \quad(p<.01)$, por lo que se considera que hay un nivel adecuado de intercorrelación para un AF.

Se condujeron dos AF. El primero fue análisis sin rotación y con el método de extracción de máxima verosimilitud; donde se eliminaron siete ítems cuyo valor de carga factorial fue menor a .40. Posteriormente, el segundo AF se realizó con el método de máxima verosimilitud con rotación Varimax y sugirió una estructura de 4 factores que explicaron el $46.88 \%$ de la varianza total.

Después de los AF se analizaron los 36 reactivos que conformaron los 4 factores. Por el contenido de los mismos, se nominaron las siguientes sub-escalas: Conciencia Emocional Intrapersonal (CE-Intra), Uso Prosocial (UPS), Conciencia Emocional Interpersonal (CE-Inter) y Uso Antisocial (UAS).

El siguiente paso consistió en analizar la fiabilidad de cada sub-escala a través del método del Alfa de Cronbach. Se igualó el número de ítems de cada sub-escala a partir de la eliminación de reactivos que disminuyeran los valores de fiabilidad de cada sub-escala por debajo de lo recomendable. La fiabilidad total final del instrumento alcanzó un Alfa de Cronbach de .87. Por su parte, las fiabilidades de cada sub-escala indicaron buenos niveles de consistencia interna: CE-Intra $(\alpha=.83)$, UPS $(\alpha=.82)$, CE-Inter $(\alpha=.72)$ y UAS (=.85).

La escala final se compuso de 24 ítems, seis por cada sub-escala, y se denominó EYUPIE-M (Escala Yucatán del Uso Percibido de la Inteligencia Emocional en Menores). Un nuevo AF sugirió una varianza total explicada de $54.53 \%$ 
Tabla 1. Carga factorial de cada ítem final resultante del AF exploratorio (>.40), media (rango de 1 a 3), desviación tipo (DT), asimetría (As) y kurtosis (K) de la EYUPIE-M

\begin{tabular}{|c|c|c|c|c|c|c|c|c|c|}
\hline & $\begin{array}{l}\text { Carga de cada factor del Análisis exploratorio, } \\
\text { Varimax, representan }>0.4\end{array}$ & CE-Intra & UPS & $\begin{array}{l}\text { CE- } \\
\text { Inter }\end{array}$ & UAS & $M$ & $D T$ & As & $K$ \\
\hline 5 & ¿Tú sabes cuándo te sientes bien? & .70 & & & & 2.54 & .70 & -1.21 & .04 \\
\hline 25 & ¿Tú sabes cuándo te sientes mal? & .65 & & & & 2.51 & .71 & -1.12 & -.18 \\
\hline 7 & ¿Te gusta cuando estás bien? & .63 & & & & 2.72 & .63 & -2.07 & 2.74 \\
\hline 4 & Cuando te sientes bien, ¿Piensas en cosas buenas? & .61 & & & & 2.64 & .66 & -1.6 & 1.18 \\
\hline 19 & ¿Te das cuenta cuando te empiezas a sentir bien? & .58 & & & & 2.51 & .71 & -1.11 & -.08 \\
\hline 23 & ¿Te das cuenta cuando dejas de sentirte mal? & .58 & & & & 2.37 & .71 & -.68 & -.79 \\
\hline 16 & $\begin{array}{l}\text { Cuando tus amigos se sienten bien, ¿Haces que se } \\
\text { sigan sintiendo bien? }\end{array}$ & & .61 & & & 2.59 & .65 & -1.31 & .47 \\
\hline 21 & $\begin{array}{l}\text { ¿Puedes hacer que tu amigo se sienta bien, para que } \\
\text { disfrute más lo que hace? }\end{array}$ & & .59 & & & 2.47 & .67 & -.88 & -.38 \\
\hline 27 & $\begin{array}{l}\text { ¿Cuándo tus amigos se sienten bien, piensan en cosas } \\
\text { buenas? }\end{array}$ & & .51 & & & 2.41 & .70 & -.77 & -.65 \\
\hline 12 & ¿Tú haces que tus amigos se sientan bien? & & .46 & & & 2.32 & .64 & -.39 & -.69 \\
\hline 41 & $\begin{array}{l}\text { Cuando es necesario, ¿Haces que tu amigo se sienta } \\
\text { bien? }\end{array}$ & & .58 & & & 2.49 & .68 & -.98 & -.28 \\
\hline 42 & $\begin{array}{l}\text { ¿Puedes hacer que tu amigo recuerde algo bueno, si } \\
\text { haces que se sienta bien? }\end{array}$ & & .56 & & & 2.43 & .71 & -.84 & -.59 \\
\hline 39 & $\begin{array}{l}\text { Después de que tu amigo se sienta bien, ¿sabes cómo } \\
\text { se va a sentir? }\end{array}$ & & & 63 & & 1.86 & .74 & .23 & -1.17 \\
\hline 35 & $\begin{array}{l}\text { Después de que tu amigo se siente mal, ¿sabes cómo } \\
\text { se va a sentir? }\end{array}$ & & & .59 & & 1.80 & .73 & .33 & -1.08 \\
\hline 24 & $\begin{array}{l}\text { ¿Tú sabes qué piensan tus amigos cuando se sienten } \\
\text { mal? }\end{array}$ & & & .53 & & 1.66 & .69 & .57 & -.80 \\
\hline 33 & ¿Sabes cuándo tus amigos dejan de sentirse bien? & & & .52 & & 2.01 & .69 & -.01 & -.87 \\
\hline 3 & Cuando tu amigo se siente mal, ¿Sabes lo que siente? & & & .48 & & 1.98 & .70 & .03 & -.96 \\
\hline 1 & $\begin{array}{l}\text { ¿Tú sabes qué piensan tus amigos cuando se sienten } \\
\text { bien? }\end{array}$ & & & .45 & & 1.82 & .68 & .24 & -.87 \\
\hline 13 & ¿Te gusta cuando tu amigo está mal? & & & & .59 & 1.31 & .67 & 1.91 & 1.95 \\
\hline 20 & $\begin{array}{l}\text { Cuando tus amigos se sienten mal, ¿Haces que se } \\
\text { sigan sintiendo mal? }\end{array}$ & & & & .58 & 1.35 & .70 & 1.71 & 1.21 \\
\hline 43 & ¿Tú haces que tus amigos se sientan mal? & & & & .56 & 1.43 & .69 & 1.31 & .33 \\
\hline 15 & $\begin{array}{l}\text { ¿Puedes hacer que tu amigo se sienta mal, para que } \\
\text { disfrute menos lo que hace? }\end{array}$ & & & & .56 & 1.37 & .69 & 1.58 & .95 \\
\hline 32 & $\begin{array}{l}\text { Cuando es necesario, ¿Haces que tu amigo se sienta } \\
\text { mal? }\end{array}$ & & & & .52 & 1.51 & .73 & 1.04 & -.35 \\
\hline 28 & $\begin{array}{l}\text { ¿Puedes hacer que tu amigo recuerde algo malo, si } \\
\text { haces que se sienta mal? }\end{array}$ & & & & .42 & 1.61 & .76 & .78 & -.85 \\
\hline
\end{tabular}

Nota.: Escala Yucatán de Uso Percibido de la Inteligencia Emocional en Menores (EYUPIE-M); Conciencia Emocional Intrapersonal (CE Intra), Uso Prosocial (UPS), Conciencia Emocional Interpersonal (CE Inter) y Uso Antisocial (UAS). Media $(M e d)$, Desviación Típica $(D t)$, Asimetría $(A)$, Kurtosis $(K)$

y una evaluación de legibilidad a través del inflesz v.1.0 reporta un Índice Flesch-Szigriszt de legibilidad de $97.46 \%$, para esta evaluación dicha puntuación es considerada de legibilidad excelente

La Tabla 1 recoge los ítems de cada subescala, muestra los 24 ítems finales con su carga factorial y sus principales descriptivos.

El siguiente objetivo era conocer la relación entre esta prueba $y$ otros instrumentos consolidados. En la Tabla II, en la cual se observa las correlaciones que se dieron entre los factores de la EYUPIE-M, con las pruebas PANAS que incluye Afecto Positivo (AP), Afecto Negativo (AN) y el Balance (B), el TMMS24 que incluye Atención (At), Claridad (Cl) y Reparación (Re) y el ESCQ incluye Percepción y comprensión emocional (PCM), Expresión y etiquetado de las emociones (EEE) y Manejo y regulación de las emociones (MRE).

Los resultados indican que la sub-escala CEIntra presentó correlaciones significativas y positivas de un tamaño de efecto entre pequeño y mediano con las tres sub-escalas del TMMS24. De forma similar, UPS se correlacionó positiva y significativamente con las tres-sub escalas del TMMS24, presentando tamaños de efecto entre pequeños y medianos. Adicionalmente, la misma sub-escala se correlacionó positiva y significativamente con las tres sub-escalas de ESCQ, con tamaños de efecto entre medianos y grandes. Por su parte, CE-Inter se correlacionó significativa y positivamente con las tres subescalas del TMMS24, y presentó tamaños de 
Tabla 2. Matriz de fiabilidad y correlaciones inter-factor del EYUPIE-M y con las sub-escalas del PANAS, TMMS24 y el ESCQ

\begin{tabular}{|c|c|c|c|c|c|c|c|c|c|c|c|c|c|c|}
\hline & $\begin{array}{c}\alpha \text { de } \\
\text { Cronbach }\end{array}$ & $n$ & CE-Intra & UPS & CE-Inter & UAS & At & $\mathrm{Cl}$ & $\mathrm{Re}$ & AP & $\mathrm{AN}$ & B & PCE & EEE \\
\hline \multicolumn{15}{|c|}{ EYUPIE-M } \\
\hline CE-Intra & .83 & 693 & . & & & & & & & & & & & \\
\hline UPS & .82 & 693 & $.64 * *$ & $\cdot$ & & & & & & & & & & \\
\hline CE-Inter & .72 & 693 & -.06 & .07 & . & & & & & & & & & \\
\hline UAS & .85 & 693 & $-.64 * *$ & $-.64 * *$ & $.30 * *$ & . & & & & & & & & \\
\hline \multicolumn{15}{|l|}{ TMMS24 } \\
\hline At & .80 & 596 & $.14^{* *}$ & $.14^{* *}$ & $.11^{* *}$ & -.04 & & & & & & & & \\
\hline $\mathrm{Cl}$ & .81 & 596 & $.20^{* *}$ & $.17^{* *}$ & $.20^{* *}$ & -.06 & $.45 * *$ & & & & & & & \\
\hline $\mathrm{Re}$ & .81 & 596 & $.21^{* * *}$ & $.19^{* *}$ & $.09^{*}$ & $-.12^{* *}$ & $.36^{* *}$ & $.58 * *$ & & & & & & \\
\hline \multicolumn{15}{|l|}{ PANAS } \\
\hline $\mathrm{AP}$ & .76 & 78 & -.03 & -.09 & -.12 & -.00 & .01 & -.06 & $-.23 *$ & . & & & & \\
\hline $\mathrm{AN}$ & .84 & 78 & -.01 & -.06 & -.13 & -.03 & -.03 & -.10 & $-.24 *$ & $.98 * *$ & . & & & \\
\hline Balance & - & 78 & -.11 & -.14 & .03 & .12 & .21 & .16 & -.01 & $.36 * *$ & .15 & . & & \\
\hline \multicolumn{15}{|l|}{ ESCQ } \\
\hline PCE & .70 & 78 & .20 & $.42^{* *}$ & .18 & -.20 & .21 & $.44 * *$ & .19 & -.08 & -.07 & -.08 & . & \\
\hline EEE & .69 & 78 & .20 & $.35^{* *}$ & .16 & -.18 & $.25^{*}$ & $.46 * *$ & $.25 *$ & -.04 & -.05 & .01 & $.74 * *$ & \\
\hline MRE & .69 & 78 & .18 & $.39^{* *}$ & .22 & $-.23^{*}$ & $.27 *$ & $.53 * *$ & $.30 * *$ & -.07 & -.09 & .08 & $.77 * *$ & $.75 * *$ \\
\hline \multicolumn{15}{|c|}{$\begin{array}{l}\text { Nota. EYUPIE-M: Conciencia Emocional Intrapersonal (CE Intra), Uso Prosocial (UPS), Conciencia Emocional Interpersonal (CE Inter) y Uso } \\
\text { Antisocial (UAS); PANAS: Afecto Positivo (AP), Afecto Negativo (AN) y el Balance (B); TMMS24: Atención (At), Claridad (Cl) y Reparación } \\
\text { (Re) y el ESCQ: Percepción y comprensión emocional (PCM), Expresión y etiquetado de las emociones (EEE) y Manejo y regulación de las } \\
\text { emociones (MRE). } \\
{ }^{*} p \leq .05 ; * * \leq .01\end{array}$} \\
\hline
\end{tabular}

efecto pequeños. Finalmente, se observó que UAS se correlacionó negativa y significativamente con una sub-escala del TMMS24 y una del ESCQ, con tamaños de efecto de pequeños a medianos.

\section{Discusión}

En cuanto al primer objetivo, referente a hacer un nuevo análisis a una medida de la IE (EIIE, Escala Infantil de la IE, Sosa-Correa \& Rodriguez-Ake, 2011), concluyó con una nueva escala de 24 ítems y cuatro sub-escalas de seis ítems cada una. Para mejorar su representación se eliminaron aquellos ítems que cargaban por debajo de 0.4 (ver tabla I) y se ajustó a una adecuada fiabilidad tanto para el valor total de la EYUPIE-M como para las fiabilidades de cada sub-escala que indicaron buenos niveles de consistencia interna como la CE-Intra, el UPS, la CE-Inter y el UAS. Dado el escaso número de ítems por sub-escala consideramos que la EYUPIE-M es una escala fiable y ajustada desde el punto de vista psicométrico.

Se consideró ponerle de nombre a la prueba final "Escala Yucatán del Uso Percibido de la Inteligencia Emocional en Menores" (EYUPIEM), ya que es un instrumento que explora en los menores de edad (entre 9 y 17 años), cómo perciben el uso que le dan a su IE según los factores que se obtuvieron, lo cuales pueden ser interpretados de la siguiente manera:

- Conciencia Emocional Intrapersonal (CEIntra), teniendo en cuenta que en este factor se agrupan preguntas como: “¿Tú sabes cuándo te sientes bien?" o “¿Tú sabes cuándo te sientes mal?", se le otorgó este nombre dada la definición de Bisquerra y Pérez Escoda (2007) del concepto Conciencia Emocional como la "capacidad para tomar conciencia de las propias emociones y de las emociones de los demás, incluyendo la habilidad para captar el clima emocional de un contexto determinado" (p.70). En este factor, solo hay preguntas de esta conciencia intrapersonal, o sea de uno mismo, por lo que no abarca la conciencia global.

- Conciencia Emocional Interpersonal (CEInter) teniendo en cuenta que en este factor se agrupan preguntas como: "Después de que tu amigo se sienta bien, ¿sabes cómo se va a sentir?" o "¿Sabes cuándo tus amigos dejan de sentirse bien? Como en el caso anterior, se le otorgó el nombre dada la definición expuesta por Bisquerra y Pérez Escoda (2007) anteriormente. En este caso la conciencia es interpersonal ya que solo hay preguntas acerca de la percepción que la persona tiene de los otros y no abarca la conciencia del sí mismo.

- En el caso de la sub-escala de Uso Prosocial (UPS), se denominó de esta manera ya que aquí se aglutinan los reactivos en donde se 
busca generar bienestar a los demás como, por ejemplo: "Cuando tus amigos se sienten bien, ¿Haces que se sigan sintiendo bien?” y “¿Puedes hacer que tu amigo se sienta bien, para que disfrute más lo que hace?". Como vimos en la introducción, la mayoría de los trabajos en adolescentes sobre la IEP predecían bienestar mental y social de forma positiva.

- En cuanto al Uso Antisocial (UAS); se denominó de esta manera ya que aquí se aglutinan los reactivos que buscan generar malestar como por ejemplo: "Cuando tus amigos se sienten mal, ¿haces que se sigan sintiendo mal?" o “¿Puedes hacer que tu amigo se sienta mal, para que disfrute menos lo que hace?" inicialmente, este factor podría estar relacionado con la tríada oscura de la personalidad (maquiavelismo, narcisismo y psicopatía subclínica) ya que según Paulhus y Williams (2002), esta triada influye negativamente en las relaciones sociales, o lo que es lo mismo usan, desde su punto de vista, su IE con un fin no prosocial. Sin embargo en la subescala UAS los ítems están más en la línea de representar más acciones de insensibilidad emocional hacia el posible daño que se genera en el otro (Kahn, Ermer, Salovey, \& Kiehl, 2016) con la probable finalidad de manipular (maquiavelismo) a los demás para su beneficio (Austin et al., 2007). Por lo que podría estar conectado sólo con la parte maquiavélica de estos denominados rasgos oscuros de la personalidad.

Referente a las correlaciones entre las subescalas del EYUPIE-M (véase Tabla II) encontramos una relación positiva significativa y moderadamente baja entre CE-inter y UAS. De alguna manera, las personas que usan sus capacidades con fines antisociales son conscientes de ello. La relación muestra que ambos factores son independientes conceptualmente, porque su relación es moderadamente baja. Sin embargo, esta relación parece darse sólo cuando el uso de la IE tiene un fin antisocial, no cuando el fin es prosocial. Lo cual concuerda con el hallazgo de relaciones positivas significativas entre índices de psicopatía y el factor de sociabilidad (Petrides et al., 2011). De acuerdo con Wai y Tiliopoulos (2012), las personas que puntúan alto en los rasgos oscuros de la personalidad mantienen su empatía cognitiva pero no la afectiva. Es decir, son conscientes de sus manipulaciones o del uso antisocial de su IE; sin embargo, no empatizan afectivamente con los demás. Por lo que parecen tener capacidad de leer y evaluar las emociones de otros, para posteriormente, utilizar esta información para estrategias con las que se pueden adquirir ventajas sobre los otros, haciéndoles pasar por alto o ignorar el potencial daño infligido a otros en el proceso (Petrides et al., 2011) para simpatizar afectivamente con los demás (Wai \& Tiliopoulos, 2012). Por consiguiente, las personas con elevado nivel de maquiavelismo, psicopatía o narcisismo tienden a ser más propensas a la manipulación de las emociones de otros para obtener un beneficio propio (Nagler, Reiter, Furtner, \& Rauthmann, 2014) y a usar tácticas de influencia interpersonal en su propio beneficio (Jonason et al., 2009, 2012; Jonason, Slomski et al., 2012;). Por lo que podemos concluir que esta correlación positiva entre ser consciente en situaciones interpersonales con el uso antisocial de la IE tiene sentido a tenor de que compartimos resultados similares con otros estudios.

También aparecieron relaciones significativas y moderadamente altas entre CE-Intra y UPS, de forma positiva y de la misma magnitud pero negativa con la UAS. Lo cual puede indicar que los evaluados en la medida que perciben que tienen más conciencia emocional de sí mismos (intrapersonal) también parecen más propensos a usar sus capacidades emocionales para un uso prosocial y una clara tendencia a evitar usar dichas capacidades de manera antisocial. Por ejemplo, cuando un menor sabe cómo se relacionan su sentimiento de bienestar con sus pensamientos y cómo se da la transición a sentirse bien, pareciera que ello le anima a compartir esa habilidad con otros para generalizar el bienestar. A su vez, cuando se percibe así mismo con poca conciencia emocional intrapersonal tenderá a ser más antisocial con los demás. Trabajos previos tanto de la IE como rasgo Petrides y cols. (2004) como de la IE como capacidad y de Mayer, Perkins y Caruso (2001) mostraron que quienes poseen mayores puntuaciones en IE son menos propensos a realizar conductas antisociales, agresivas o de acoso a otros compañeros que aquellos que puntúan menos en IE. Estas tres subescalas CE-intra, UPS y UAS mostraron correlaciones moderadamente altas entre sí, lo que 
parece indicar la presencia de casualidad, si bien dichos factores parecen ser independientes entre sí.

El segundo objetivo fue demostrar si otros instrumentos ya consolidados de IEP como el TMMS-24 y el ESCQ, así como el PANAS, están correlacionados con los factores de la nueva medida EYUPIE-M.

En cuanto al TMMS-24, se observan correlaciones positivas de los tres factores (At, $\mathrm{Cl}$ y Re) con la CE-Intra, con el UPS y con CE-Inter. En el sentido de que los adolescentes que se perciben con altas puntuaciones en estos factores también muestran altos niveles de atención, claridad y reparación emocional, lo cual concuerda con la tradición positiva que a mayor IEP mayor es la tendencia prosocial y conciencia emocional (Pedrosa et al., 2014). Sin embargo, si hay relación significativa esto no parece indicar que en futuros análisis de regresión dichas vinculaciones permanezcan. Por lo que el TMMS24 podría no poder explicar el porqué del uso social o antisocial de la IE. Lo único que parece indicar que aquéllos que perciben una mayor IEP también tienden a percibirse con mejor conciencia emocional y también piensan que lo usan con fines adecuados.

También se observa una correlación significativa, negativa y baja entre el UAS de la EAYUPIE-M y la Re del TMMS-24, lo cual indica que en la medida que los jóvenes se perciban con la capacidad de generar malestar en los otros, se perciben con menos capacidad de regular sus emociones, lo cual concuerda con lo encontrado en algunos estudios (Hayes \& O’Reilly, 2013; Kahn et al., 2016; Wai \& Tiliopoulos, 2012). Tal parece que el no poder regular sus propias emociones hace que deseen $o$ se interesen más por el malestar de los demás que por aprender a gestionar sus emociones para un mejor funcionamiento social (Mestre, GutiérrezTrigo, Guerrero, \& Guil, 2017).

En cuanto a las correlaciones entre la EYUPIE-M y el ESCQ, se observa que sólo la sub-escala UPS correlaciona de manera positiva entre baja y moderada con las tres sub-escalas del ESCQ. Lo que implica que a mayor percepción de que la persona puede ayudar a sentirse bien a otros, mayor percepción y comprensión; expresión y etiquetado; y regulación emocional parece percibir que tienen. Este resultado es similar a los encontrados por Costa y Faria (2015). Por otro lado se observa una correlación negativa baja entre UAS y la MRE, lo cual indica que en la medida que una persona se percibe con la habilidad de generar malestar a otros, más dificultad cree tener para gestionar sus emociones. Lo cual concuerda la correlación antes mencionada entre el UAS y la Re del TMMS-24. El hecho de hacer sentir mal a los demás parece indicar que ellos se perciben con menos IEP, algo ya contrastado (Casas, Ortega-Ruiz, \& Del Rey, 2015; Pérez-Villalobos, Jiménez-Espinoza, VilosNúñez, \& Ortíz-Véliz, 2015). Otros estudios han mostrado que una mejor capacidad para la IE, no percibida sino real, se relaciona con una menor participación en conductas antisociales como las conductivas disruptivas (Mayer et al., 2001).

No se encontraron relaciones entre los cuatro factores de la EYUPIE-M con el AP ni con el AN del PANAS, lo cual parece indicar que independientemente que los menores se consideren conscientes de sus emociones, y de la de los demás, que perciban su apoyo hacia los demás, o que sean capaces de generar malestar, ninguno de estos cuatro usos, parecen estar relacionados con su estado afectivo, positivo, negativo, ni con el balance de ambos estados emocional, por lo que ni percibirse capaz de hacer el bien implica sentirse con afecto positivo o negativo, así como tampoco percibirse como capaz de hacer el mal implica que la persona se sienta con afecto positivo o negativo: Lo cual contradice lo encontrado por Dufey y Fernandez (2012) quienes muestran que la generación de malestar hacia otros está significativamente correlacionado con el Afecto Negativo y en correlación inversa con AP. Pero pudiera explicarse por lo hallado en Gutiérrez-Cobo y cols., (2016) en cuanto a que las pruebas de autoreporte no están relacionadas con las tareas cognitivas Hot ni Cool. En este caso puede estar representado por el estado de ánimo de los evaluados, de tal forma que la carga emocional del joven no influye en la percepción del uso que le da a su IE.

Una vez analizados los dos objetivos planteados en esta investigación, se observan otras dos conclusiones relevantes, la primera es contar con un factor antisocial en una escala de IE que 
permite observar cómo esta capacidad percibida está relacionada con la autoeficacia emocional, por lo que hace pensar que las personas que saben más de los procesos emocionales de los otros (empatía cognitiva), se perciben con mayor capacidad de hacer daño a los demás, como mencionaron otros trabajos que encontraron correlación positiva de la generación de daño con la capacidad de ser socialmente agradables (Nagler et al., 2014; Petrides et al., 2011; Wai \& Tiliopoulos, 2012).

Otra conclusión, es que este instrumento ha sido creado para evaluar la IEP a través de una combinación del uso que se le da a la IE junto con IE rasgo, mostrando evidencia de validez. Considerando además que esta prueba es para muestras heterogéneas como los menores de edad en centros escolares con apenas filtros selectivos, lo que nos permitirá entender qué uso hacen de sus capacidades. En conclusión se observa que a pesar de que parece una escala prometedora hay evidentes limitaciones en este trabajo como las que se mencionan a continuación.

El nivel de respuesta de la escala Likert de la EYUPIE-M debería aumentar de tres opciones a, al menos, 5 o 7, para una mejor observación de diferencias individuales y sus relaciones con otras variables. Este reporte analiza diversas aplicaciones en las que algunas pruebas se obtuvo un número reducido de participantes, por lo que se propone hacer estudios en general con mayor cantidad de jóvenes, no solo en la aplicación de la EYUPIE-M sino también en las pruebas que sirvan para corroborar la validez convergente y divergente, como pueden ser pruebas que midan tanto la empatía como la triada oscura de la personalidad.

A partir de este estudio, la perspectiva a futuro que se considera relevante, además de subsanar las limitaciones anteriormente descritas, es investigar, si este instrumento que incluye IE rasgo y el uso que le dan a esta, predice criterios de regulación emocional, particularmente si hay una interacción entre IE Rasgo con IE Capacidad con la finalidad de servir de screening para aquéllos sujetos con ideas antisociales.

\section{Referencias}

Austin, E. J., Farrelly, D., Black, C. \& Moore, H.
(2007).

Emotional

Machiavellianism and

intelligence, manipulation: Does EI have a dark side? Personality and Individual Differences, 43(1), 179-189.

https://doi.org/10.1016/j.paid.2006.11.019

Barchard, K. A., Brackett, M. A. \& Mestre, J. M. (2016). Taking Stock and Moving Forward: 25 Years of Emotional Intelligence Research. Emotion Review, 8(4), 303-312. https://doi.org/10.1177/1754073916650562

Bisquerra, R. \& Pérez Escoda, N. (2007). Las competencias emocionales. Educación XX1, 10 ,

$61-82$.

https://doi.org/10.5944/educxx1.1.10.297

Casas, J. A., Ortega-Ruiz, R. \& Del Rey, R. (2015). Bullying: The impact of teacher management and trait emotional intelligence. The British Journal of Educational Psychology, 85(3), 407-23. https://doi.org/10.1111/bjep.12082

Costa, A. \& Faria, L. (2015). The impact of emotional intelligence on academic achievement: A longitudinal study in Portuguese secondary school. Learning and Individual Differences, 37, 38-47. https://doi.org/10.1016/j.lindif.2014.11.011

Díaz-Castela, M., Hale, W., Muela, J. A., Espinosa-Fernández, L., Klimstra, T. \& Garcia-Lopez, L. J. (2013). La medición de la Inteligencia Emocional en adolescentes españoles con trastorno de ansiedad. Anales de Psicología, 29(2), 509-515. https://doi.org/10.6018/analesps.29.2.144271

Dufey, M. \& Fernandez, A. (2012). Validez y confiabilidad del Positive Affect and Negative Affect Schedule ( PANAS ) en estudiantes universitarios chilenos. Revista Iberoamericana de Diagnóstico Y Evaluación Psicológica, 1, 157-173. Retrieved from http://www.aidep.org/03_ridep/R34/ART 8.pdf

Extremera, N., Fernández-Berrocal, P. \& Salovey, P. (2006). Spanish version of the MayerSalovey-Caruso Emotional Intelligence Test (MSCEIT). Version 2.0: Reliabilities, age and gender differences. Psicothema, 18(suplemento), 42-48. https://doi.org/10.1037/1528-3542.3.1.97

Faria, L., Santos, N. L., Takšić, V., Räty, H., 
Molander, B., Holmström, S. \& Toyota, H. (2006). Cross-Cultural Validation of the Emotional Skills and Competence Questionnaire (Escq). Psicología, XX(2), 95127.

Fariselli, L., Ghini, M. \& Freedman, J. (2008). Age and Emotional Intelligence. Six Seconds: The Emotional Intelligence Network, 1-10.

Fernández-Berrocal, P., Alcaide, R., Domínguez, E., Fernández-McNally, C., Ramos, N. S. \& Ravira, M. (1998). Adaptación al castellano de la escala rasgo de metaconocimiento sobre estados emocionales de Salovey et al.: datos preliminares. Libro de Actas Del V Congreso de Evaluación Psicológica., 1, 83-84.

Fernández-Berrocal, P. \& Extremera, N. (2005). La inteligencia emocional como una habilidad esencial en la escuela. Revista Electrónica Iberoamericana de Educación, 29, 1-6.

Fernandez-Berrocal, P., Extremera, N. \& Ramos, N. (2004). Validity and reliability of the Spanish modified version of the Trait MetaMood Scale. Psychological Reports, 94, 751755. https://doi.org/10.2466/PR0.94.3.751755

Gutentag, T., Halperin, E., Porat, R., Bigman, Y. E. \& Tamir, M. (2016). Successful emotion regulation requires both conviction and skill: beliefs about the controllability of emotions, reappraisal, and regulation success. Cognition and Emotion, 9931(August), 1-9. https://doi.org/10.1080/02699931.2016.12137 04

Gutiérrez-Cobo, M. J., Cabello, R. \& FernándezBerrocal, P. (2016). The Relationship between Emotional Intelligence and Cool and $\mathrm{Hot}$ Cognitive Processes: A Systematic Review. Frontiers in Behavioral Neuroscience, 10(101), 1-13. https://doi.org/10.3389/fnbeh.2016.00101

Hayes, J. M. \& O’Reilly, G. . (2013). Psychiatric disorder, IQ, and emotional intelligence among adolescent detainees: A comparative study. Legal and Criminological Psychology, 18(1), 30-47. https://doi.org/10.1111/j.20448333.2011.02027.x

Jiménez, I. \& López Zafra, E. (2011). Actitudes sociales y adaptación social en adolescentes españoles: el papel de la inteligencia emocional percibida. Revista de Psicología
Social, 26(1), 105-117. https://doi.org/10.1174/021347411794078417 Jonason, P. K., Li, N. P., Webster, G. D. \& Schmitt, D. P. (2009). The Dark Triad: Facilitating a short-term mating strategy in men. European Journal of Personality. https://doi.org/10.1002/per.698

Jonason, P. K., Slomski, S. \& Partyka, J. (2012). The Dark Triad at work: How toxic employees get their way. Personality and Individual Differences, 52(3), 449-453. https://doi.org/10.1016/j.paid.2011.11.008

Jonason, P. K., Webster, G. D., Schmitt, D. P., Li, N. P. \& Crysel, L. (2012). The antihero in popular culture: Life history theory and the dark triad personality traits. Review of General Psychology, 16(2), 192-199. https://doi.org/10.1037/a0027914

Kahn, R. E., Ermer, E., Salovey, P. \& Kiehl, K. A. (2016). Emotional Intelligence and Callous???Unemotional Traits in Incarcerated Adolescents. Child Psychiatry and Human Development, 47(6), 1-15. https://doi.org/10.1007/s10578-015-0621-4

Lopes, P. N. (2016). Emotional Intelligence in Organizations: Bridging Research and Practice. Emotion Review, 8(4), 316-321. https://doi.org/doi/pdf/10.1177/175407391665 0496

Mayer, J. D., Caruso, D. R. \& Salovey, P. (2016). The ability model of emotional intelligence: Principles and updates. Emotion Review, 8(4), 290-300. https://doi.org/10.1177/1754073916639667

Mayer, J. D., Perkins, D. M., Caruso, D. R. \& Salovey, P. (2001). Emotional Intelligence and Giftedness. Roeper Review, 23(3), 131137. Retrieved from http://www.unh.edu/emotional_intelligence/EI Assets/EmotionalIntelligenceProper/EI2001Gi ftedness.pdf

Mayer, J. D. \& Salovey, P. (1997). What is emotional intelligence? Emotional Development and Emotional Intelligence. https://doi.org/10.1177/1066480710387486

Mestre, J. M., Guil, R., Rodriguez-Cordon, J., Pérez-Conzález j. \& Cejudo, J. (2015). Assessing Emotional Intelligence As Criteria: Theoretical and Research Implications. BiAnnual Conference of the International 
Society for Research on Emotion, 2015. Retrieved from http://www.isre2015.org/sites/default/files/Me stre.pdf\#overlay-context=node/87

Mestre, J. M., MacCann, C. \& Roberts, R. D. (2016). Models of Cognitive Ability and Emotion Can Better Inform Contemporary Emotional Intelligence Frameworks. Emotion Review. https://doi.org/10.1177/1754073916650497

Mestre, J. M., Núñez-Vázquez, I. \& Guil, R. (2007). "Aspectos psicoevolutivos, psicosociales y diferenciales de la inteligencia emocional." In Piramide (Ed.), Manual de Inteligencia Emocional. (p. 153-171.). Madrid.

Nagler, U. K. J., Reiter, K. J., Furtner, M. R. \& Rauthmann, J. F. (2014). Is there a "dark intelligence"? Emotional intelligence is used by dark personalities to emotionally manipulate others. Personality and Individual Differences, 65, 47-52. https://doi.org/10.1016/j.paid.2014.01.025

Nathanson, L. Rivers, S., E. Flynn L., M. \& Brackett M., A. (2016). Creating Emotionally Intelligent Schools with RULER. School Administrator, 73(4), 19-22. https://doi.org/10.1177/1754073916650495

Paulhus, D. \& Williams, K. (2002). The dark triad of personality: Narcissism, Machiavellianism, and psychopathy. Journal of Research in Personality. Retrieved from http://www.sciencedirect.com/science/article/ pii/S0092656602005056

Pedrosa, I., Suarez-Alvarez, J., Lozano, L. M., Muñiz, J. \& Garcia-Cueto, E. (2014). Assessing Perceived Emotional Intelligence in Adolescents: New Validity Evidence of Trait Meta-Mood Scale-24. Journal of Psychoeducational Assessment, 32(8), 737-746. https://doi.org/10.1177/0734282914539238

Pérez-Villalobos, C., Jiménez-Espinoza, J., VilosNúñez, V. \& Ortíz-Véliz, H. (2015). Análisis de las Propiedades Psicométricas de la Escala de Satisfacción con la Gestión Escolar para Padres. Revista Iberoamericana de Diagnóstico y Evaluación, 1(39), 34-45. Retrieved from http://www.aidep.org/03_ridep/R39/Art3.pdf Petrides, K. V. (2016). Four Thoughts on Trait
Emotional Intelligence. Emotion Review, 8, 650504. https://doi.org/10.1177/1754073916650504

Petrides, K. V, Vernon, P. a, Schermer, J. A. \& Veselka, L. (2011). Trait emotional intelligence and the dark triad traits of personality. Twin Research and Human Genetics: The Official Journal of the International Society for Twin Studies, 14(1), 35-41. https://doi.org/10.1375/twin.14.1.35

Roberts, R. D., MacCann, C., Guil, R. \& Mestre, J. M. (2016). Reimagining Emotional Intelligence : A Healthy, Much Needed, and Important Progression for the Field. Emotion $R, \quad 8(4), \quad 0-0$. https://doi.org/10.1177/1754073916650506

Salovey, P. \& Mayer, J. D. (1990). Emotional intelligence. Imagination, Cognition, and Personality, 9, 185-211.

Sánchez-Álvarez, N., Extremera, N. \& FernándezBerrocal, P. (2016). The relation between emotional intelligence and subjective wellbeing: A meta-analytic investigation. The Journal of Positive Psychology, 11(3), 276(3), 276-285.

https://doi.org/http://dx.doi.org/10.1080/1743 9760.2015.1058968

Sandín, B., Chorot, P., Valiente, R. M. \& Santed, Y. M. A. (1998). Frecuencia e intensidad de los miedos en los niños: Datos normativos. Revista de Psicopatología Y Psicología Clínica, 3(1), 15-25. Retrieved from http://revistas.uned.es/index.php/RPPC/article /viewFile/3853/3708

Sosa-Correa, M. \& Rodriguez-Ake, A. (2011). Inteligencia Emocional. Revista Mexicana de Psicología, Especial, 909-910.

Taksic, V., Mohoric, T. \& Duran, M. (2009). Emotional skills and competence questionnaire (ESCQ) as a self-report measure of emotional intelligence. Psiholoska Obzorja / Horizons of Psychology, 18(3), 7-21. Retrieved from http://ovidsp.ovid.com/ovidweb.cgi?T=JS\&P $\mathrm{AGE}=$ reference $\& \mathrm{D}=\mathrm{psyc} 6 \& \mathrm{NEWS}=\mathrm{N} \& \mathrm{AN}=$ 2010-02324-002

Wai, M. \& Tiliopoulos, N. (2012). The affective and cognitive empathic nature of the dark triad of personality. Personality and Individual Differences, 52(7), 794-799. https://doi.org/10.1016/j.paid.2012.01.008 